\title{
A rare case of infective endocarditis in a quadricuspid aortic valve caused by Gemella sanguinis
}

\author{
Hyun $\mathrm{Ji}^{* 1}$, Shravya Vinnakota ${ }^{2}$, Walter R. Wilson ${ }^{3}$, Vuyisile T. Nkomo ${ }^{2}$ \\ ${ }^{1}$ Department of Internal Medicine, Mayo Clinic, USA \\ ${ }^{2}$ Department of Cardiovascular Diseases, Mayo Clinic, USA \\ ${ }^{3}$ Division of Infectious Diseases, Mayo Clinic, USA
}

Received: August 11, 2020

DOI: $10.5430 /$ dcc.v7n3p17
Accepted: November 18, 2020 Online Published: January 4, 2021

URL: https://doi.org/10.5430/dcc.v7n3p17

\begin{abstract}
Infective endocarditis (IE) in patients with a quadricusp aortic valve (QAV) is a rare occurrence that has only been described nine times in English literature. Similar in rarity, IE by Gemella sanguinis has only been described ten times. It has never been described in a patient with QAV. We describe a case of Gemella sanguinis infective endocarditis of a quadricusp aortic valve in a patient in his $4^{\text {th }}$ decade of life. His QAV was detected on pre-operative transesophageal echocardiogram and confirmed intra-operatively. The patient was managed with bioprosthetic aortic valve replacement and a 6-week course of intravenous antibiotics.
\end{abstract}

Key Words: Quadricusp aortic valve, Infective endocarditis, Gemella sanguinis, Aortic regurgitatio

\section{INTRODUCTION}

Quadricusp aortic valve (QAV) is a rare congenital anomaly with an incidence $0.008 \%$ of autopsies, and $1 \%$ of aortic valve surgeries. ${ }^{[1]}$ Patients with QAV are usually diagnosed incidentally on transthoracic echocardiogram (TTE) or with severe aortic regurgitation requiring surgery in the 4 th or 5th decade of life. Otherwise, it may be completely asymptomatic and discovered incidentally on echocardiogram, surgery or autopsy. Infective endocarditis (IE) of a QAV is rarely described in literature, and is mostly associated with the common pathogens of infective endocarditis such as Streptococcus species and Enterococcus species. Our patient was diagnosed with a QAV through pre-operative transesophageal echocardiogram and intraoperatively during an aortic valve replacement for severe aortic regurgitation in the setting IE. Gemella sanguinis was recovered from blood cultures and intraoperative aortic valve cultures. G sanguis IE was reported only ten times previously.

\section{CASE REPORT}

A 48-year-old man presented with increasing fatigue and a constellation of associated symptoms including diffuse arthralgias, night sweats, subjective fevers, chills and a $10 \mathrm{lb}$ weight loss in the past 3 months. He developed a vesicular rash over his arms and chest 4 weeks prior that improved with a short course of prednisone. He was empirically prescribed levofloxacin for a progressive dry cough three days prior to presentation. Notably, he had recent travel to the Dominican Republic 3 months prior, a dental cleaning without extractions 2 months prior, and had worked with poultry for several years. His only medical history consisted of a platelet disorder called May-Hegglin syndrome. He reported two uncles diagnosed with bicuspid aortic valves and a mother

*Correspondence: Hyun Ji; Email: ji.hyun@mayo.edu; Address: Department of Internal Medicine, Mayo Clinic, USA. 
with history of an unevaluated murmur.

Vital signs revealed a temperature of $36.4^{\circ} \mathrm{C}$, heart rate of 74 beats/min, blood pressure $124 / 49 \mathrm{mmHg}$, respiratory rate of 19 breaths per minute and oxygen saturation of $97 \%$ on room air. Physical examination revealed a well appearing man with an early diastolic and decrescendo murmur along the left sternal border, clear lungs, and splinter hemorrhages on bilateral fingernails.

Initial labs were significant for a hemoglobin of $11.4 \mathrm{~g} / \mathrm{dl}$ (normal reference 13.2-16.6 g/dl), MCV 87.6 fl (78.2-97.9 fl), platelet $50,000 / \mu \mathrm{l}(135-317 / \mu \mathrm{l})$ and WBC 6,600/ $\mu \mathrm{l}(3.4$ $9.6 / \mu \mathrm{l})$ with $4.79 \times 10 / \mu \mathrm{l}$ neutrophils $(1.56-6.45 / \mu \mathrm{l})$. Creatinine and electrolytes were within normal limits. Urinalysis was unremarkable. Transthoracic echocardiogram (TTE) showed a thickened aortic valve with an indeterminate cusp number with suspected flail/torn leaflet and severe aortic regurgitation. Subsequent transesophageal echocardiography (TEE) showed a $2 \mathrm{~cm}$-vegetation and $4 \mathrm{~mm}$-vegetation on the non-coronary cusp consistent with native aortic valve endocarditis and possible bicuspid aortic valve (see Figure 1). Blood cultures were drawn and the patient was initiated on broad-spectrum antibiotics. Further work-up of possible culture-negative endocarditis was negative for Bartonella, Brucella, Coxiella and cardiolipin antibodies. Panorex was negative for any active dental infection. After 61 hours, 2 separate sets of blood cultures speciated Gemella sanguinis susceptible to ceftriaxone, vancomycin and penicillin.

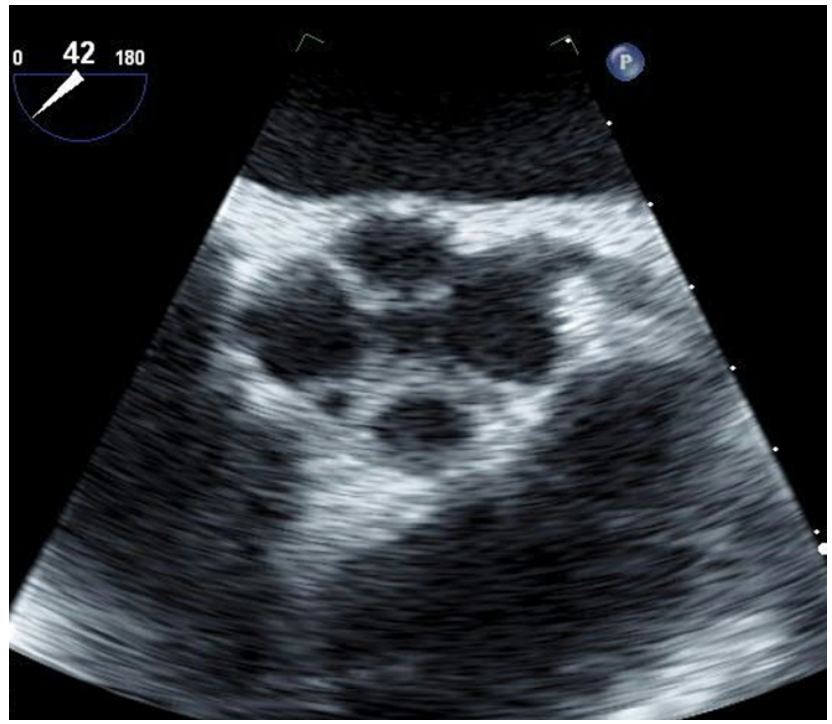

Figure 1. Quadricusp aortic valve seen on transesophageal echocardiogram

The patient was diagnosed with IE using the Modified Duke Criteria from the AHA Guidelines 2015 by fulfilling 2 major criteria of 1) 2 blood cultures $>12$ hours apart with a species known to cause IE and 2) echocardiographic evidence of endocardial involvement. He also fulfilled several minor criteria including a vascular phenomenon demonstrated by splinter hemorrhages and a predisposing heart condition seen on echocardiogram.

Antibiotics were narrowed based on susceptibilites. He underwent aortic valve replacement with a tissue valve five days after admission. Intraoperatively, he was noted to have a quadricuspid aortic valve (type B according to the Hurwitz classification) with a small extra leaflet between the noncoronary cusp and the left coronary cusp (see Figure 2). He had vegetations with perforations on the extra leaflet and noncoronary cusp. Surgical pathology showed active native valve endocarditis with cocci identified and with focal purulent inflammation and valve destruction. Intraoperative broad range PCR was positive for Gemella sanguinis. His postoperative course was largely unremarkable, and he was discharged to complete a 6 week course of IV antibiotics.

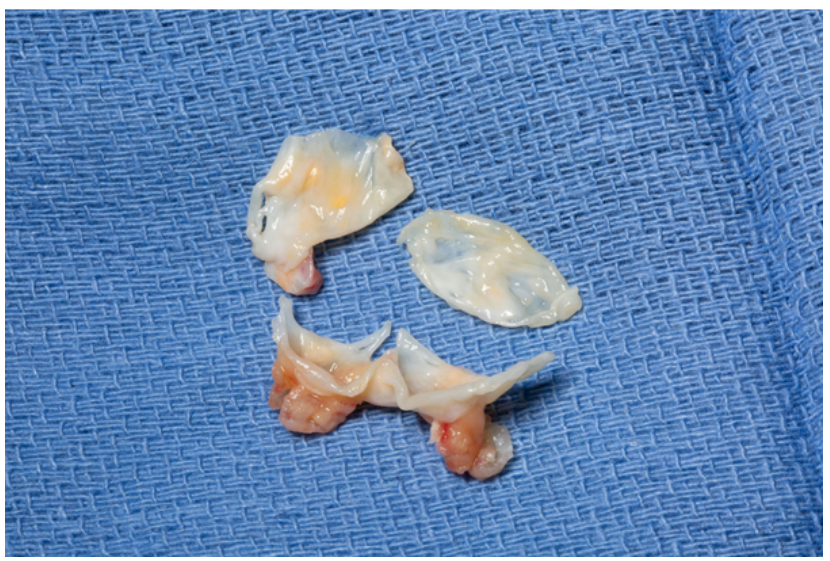

Figure 2. Quadricusp aortic valve surgical specimen

\section{DisCuSSION/CONCLUSION}

QAV is a rare phenomonem with an incidence of $0.043 \%$ in 2-D TTE and $1 \%$ of aortic valve surgeries. ${ }^{[1]}$ Hurwitz and Roberts described 7 different types of aortic valves (type A-G), with type A and B being the most common in surgical cases. $^{[2]}$ A comphrensive review of 50 QAV cases diagnosed by echocardiogram between January 1975 and March 2014 showed that most patients were diagnosed in their $4^{\text {th }}$ decade without a preference for either gender. ${ }^{[3]}$ No cases of infective endocarditis were documented during follow up of 4.8 \pm 5.6 years and only $16 \%$ of patients required aortic valve surgery with aortic regurgiation being the most common surgical indication. ${ }^{[3]}$ Because of its low incidence, the risk of infective endocarditis among patients with QAV is unknown, and it has only been described nine times in modern literature. It is hypothesized that variants with unequal cusps 
predisposes patients to infective endocarditis due to unequal stress among the valves. However, review of case reports in literature does not support this hypothesis. ${ }^{[4]}$ Previous organisms described in QAV IE include Streptococcus species, Corynebacterium striatum, and Enterococcus faecalis. ${ }^{[1,4-11]}$

The most common pathogens causing IE are Staphylococcus, Streptococcus, Enterococcus or HACEK species. ${ }^{[5]}$ Gemella sanguinis is a catalase negative gram positive cocci, usually grown in blood agar, that is a rare cause of infective endocarditis. It has been associated with poor dental health or dental procedures. ${ }^{[5]}$ Our patient did not have poor dental health, but did have a recent dental cleaning preceeding some of his symptoms. Dental procedures are not considered a risk factor for development of IE. ${ }^{[12]}$ A review of 12 cases from 1998 to 2017 showed that $58.3 \%$ (7/12) cases were associated with periodontal disease or procedures and $81.8 \%$ (9/11) cases were associated with native valves, 2 of which were bicuspid aortic valves. ${ }^{[5]}$ There is no evidence in current literature suggesting that there is an increased risk of IE in patients undergoing dental procedures and antibiotic prophylaxis is recommended only in patients with the highest risk of adverse outcome from IE. ${ }^{[3,12]}$ Gemella sanguinis has not been described in literature to be associated with a quadricusp aortic valve.

From our literature search, there is no known association between May-Hegglin syndrome and QAVs.
Given the emergence of new cardiac imaging such as magentic resonance imaging (MRI) and the increased quality of echocardiography, it is possible that we will see a higher incidence of QAV. It is unclear whether there is a genetic correlation, and there are currently no clear recommendations on whether family members should be screened. It is interesting to note that this patient's two uncles had a history of diagnosed bicuspid aortic valve, and mother with an unclassified murmur. In addition, though rare, we should consider Gemella sanguinis as a cause of infective endocarditis.

\section{STATEMENT OF ETHICS}

The patient has given informed consent to use his medical record for academic purposes.

\section{Author CONTRIBUTIONS}

$\mathrm{HJ}$ had a substantial contribution to the conception and design of the work, analysis and interpretation of the literature, and wrote a significant part of the draft. She took part in revision and final approval of the publication. SV, WRW and VTN contributed to the analysis of the literature, revising the work for important intellectual content and final approval of the version to be published.

\section{CONFlicts OF InTEREST Disclosure}

The authors have no conflicts of interest to declare.

\section{REFERENCES}

[1] Ghalem A, Bachrif M, Hbali A, et al. Type A Quadricuspid Aortic Valve Infective Endocarditis Complicated by Multiple Aortocardiac Fistulae: Case Report and Brief Literature Review. Case Reports Cardiol.

[2] Hurwitz LE, Roberts WC. Quadricuspid Semilunar Valve. 1973 May; 31(5): 623-626. https://doi.org/10.1016/0002-9149(73)90332-9

[3] Tsang M, Abudiab M, Ammash N, et al. Quadricusp Aortic Valve: Characteristics, Associated Structural Cardiovascular Abnormalities, and Clinical Outcomes. Circulation. 2016 Jan; 133(3): 312-9. https://doi.org/10.1161/CIRCULATIONAHA.115.017743 PMid:26635401

[4] Mizoguchi H, Sakaki M, Inoue K, et al. Quadricusp aortic valve complicated with infective endocarditis: report of a case. Surg Today. 2014 Dec; 44(12): 2388-2391. https://doi.org/10.1007/s00595-0140844-1 PMid:24496979

[5] Maraki S, Plevritaki A, Kofteridis D, et al. Bicuspid aortic valve endocarditis caused by Gemella sanguinis: Case report and literature review. J Infect Public Health. 2019 May; 12(3): 304-308. https://doi.org/10.1016/j.jiph.2019.01.001 PMid:30670353

[6] Matsukawa T, Yoshii S, Hashimoto R, et al. Quadricusp Aortic Valve Perforation Resulting From Bacterial Endocarditis - 2D Echo and angiographic diagnosis and its surgical treatment. Jpn Circ J. 2002 May; 52(5): 437-40. https://doi.org/10.1253/jcj.52.437 PMid:3045360

Published by Sciedu Press
[7] Finch A, Osman K, Kim KS, et al. Transesophageal Echocardiography Findings of an Infected Quadricuspid Aortic Valve with an Anomalous Coronary Artery. Echocardiography. 1994 July; 11(4): 369-375. https://doi.org/10.1111/j.1540-8175.1994.tb01379.x

[8] Watanabe Y, Taketani Y, Takei Y, et al. Complete heart block resulting from quadricuspid aortic valve penicillin-resistant pneumococcal endocarditis: a case report. Circ J. 2003 Mar; 67(3): 275-276. https://doi.org/10.1253/circj.67.275 PMid:12604882

[9] Takeda N, Ohtaki E, Kasegawa H, et al. Infective Endocarditis Associated with Quadricuspid Aortic Valve. Jpn Heart J. 2003 May; 44(3): 441-445. https://doi.org/10.1536/jhj.44.441 PMid:12825812

[10] Bauer F, Litzler PY, Tabley A, et al. Endocarditis complicating a congenital quadricuspid aortic valve. Eur J Echocardiogr. 2008 May; 9(3): 386-387. https://doi.org/10.1016/j.euje.2006.12.001 PMid:17320484

[11] Jackson C, Sarwar T, Hwang I, et al. Quadricuspid aortic valve infective endocarditis. J Clin Ultrasound. 2018 Feb; 46(2): 145-148. https://doi.org/10.1002/jcu.22495 PMid:28862762

[12] Wilson W, Taubert K, Gewitz M, et al. Prevention of infective endocarditis: guidelines from the American Heart Association: a guideline from the American Heart Association Rheumatic Fever, Endocarditis, and Kawasaki Disease Committee, Council on Cardiovascular Disease in the Young, and the Council on Clinical Cardiology, Council on Cardiovascular Surgery and Anesthesia, and the Quality of Care and Outcomes Research Interdisciplinary Working Group. Circulation. 2007 Oct; 116(15): 1736. 